ISSN 1392-1541

Geodezija ir kartografija, 2005, XXXI t., Nr. 1 Geodesy and Cartography, 2005, Vol XXXI, No 1

UDK 528.082

\title{
ANALYSIS OF DISCRETISATION STRATEGY FOR AREA AND SPACE MEASUREMENT
}

\author{
Vytautas Giniotis, Darius Mariūnas \\ Dept of Geodesy and Cadastre, Vilnius Gediminas Technical University, \\ Sauletekio al. 11, LT-10223 Vilnius-40, Lithuania, \\ e-mail:vgg@ai.vtu.lt \\ Received 1209 2004; accepted 03012005
}

\begin{abstract}
Measurement strategy is discussed in the article. It is important to optimise the data selection (sampling) from the object for the determination of its geometrical features within some limits of accuracy. The minimal and maximal intervals of measurement must be selected ensuring the maximal efficiency of operation and the accuracy as well. In machine engineering the typical case is in the calibration of coordinate measuring machines (CMMs) as it is a quite complicated task because of the variety of accuracy parameters to be checked and the high accuracy that must be assured. Some new techniques for the two- and three-dimensional measurements are discussed in this paper leading to a more efficient calibration process. This is relevant to machine engineering where geometric accuracy parameters are to be determined, to the geodetic measurements where slopes of terrain, area flatness and volumetric features are surveyed, in structural engineering, etc.
\end{abstract}

Keywords: measurement, sampling, accuracy, coordinate, calibration, geometric parameters.

\section{Introduction}

Technical problems of sampling in coordinate measurements are discussed in [1-5], etc. Sampling procedures are important in dimensional measurements, measurement of geometrical features and position deviations between them. The main approach in this procedure is probability theory giving statistical means for evaluating the results of measurement (the measurand) by selecting the pitch of measurement that serves as a kind of sampling, assessing the set of trials, the mean value of estimates, the evaluation of dispersion at the probability level chosen. It is a basis for data processing in all measurements and is widely used in all kinds and branches of metrology. During the calibration of scales [3] it is possible to find out only a restricted number of values from the full range of existing data. An example of the results shows that during the accuracy calibration some larger values of the error can be omitted including some significant ones. The problem exists due to the great amount of information that is present in the calibration of numerically-controlled machines, such as coordinate measuring machines (CMM) in their total volume. Suitable sampling of measuring points on the surface of industrial parts was shown to be a very important task, as was the sampling of the points in the machine's volume during its calibration. The same problem exists in the surveying operations [4], the difficulties being in the determination of acceptable pitch of measurement to determine the slope, curvatures, peaks and valleys of terrain adequately.

Sladek and Krawczyk [5] have highlighted the problem due to the great amount of information that is needed to calibrate a CMM using the total volume. The authors showed that it was technically difficult and economically demanding to calibrate the enormous number of points, eg, 324000 steps of the rotary table of the CMM in the measuring volume arising from six rotary axes. Suitable sampling of measuring points on the surface of industrial parts is a very important task as was the sampling of the points in the CMM volume during its calibration. The accuracy of calibration must remain at the same level although the time consumed must be minimised.

Sampling strategy for dimensional measurement of geometric features on the CMM was discussed by Lee et al [2]. The Hammersley sequence and a stratified sampling method were used by the authors for assessing geometric accuracy of the workpiece depending on its manufacturing process and dimensions. Investigations showed that the Hammersley sequence had a nearly quadratic reduction in the number of points to be checked when using the random or the uniform sampling method. The sampling strategy for various geometric features of parts measurement in industry is especially important in computer-integrated manufacturing where both time and cost are significant.

Various measurement models and methods are used for sampling strategy during the selection of the optimal number of points in the measuring volume. It is widely analysed in several research works [6-10]. Succession models as Hamersley, Halton - Zaremba are used in which the coordinates of measurement points are calculated according to particular formulas for the flatness and volume measurements. 
Woo et al, Yau et al $[11,12]$ propose a hierarchyplanned system for the determination of errors using CMM. This allows selecting the measurement process and the coordinates for the measurement of errors to be selected. Some authors use the "grey" theory [8] to forecast measurement points, B - splines and other models of parameter modelling $[9,10]$. The most analysed methods are associated with the set of mathematical models, generated series or splines. The coordinates of the measurement errors often depend on the type of spline, and if it is to be changed, then another set of coordinates will be generated. The area, where errors vary evenly, the pitch of discretisation can be chosen larger and that, where variation is quite sharp significantly less [10]. The methods discussed above are connected with mathematical models based on sequences or the generation of splines. The measurement strategy is strongly dependant on the method of splitting the area or volume that is to be measured.

Having in mind many varieties of information assessment during the measurement, it is evident that the sampling problem is important and is worthwhile to develop. The most evident example used in all fields of metrology is calibration of weights. The scale of electronic weights cannot be calibrated at every $0,1 \mathrm{mg}$ or so. A calibration process is carried out at some pitch of the scale range. Thus, some intervals between the calibrated points on the scale are left undetermined.

The purpose of this work is the analysis of the possibilities of discretisation of two- and threedimensional space, evaluating the trend of the function of the systematic error gradient in the $\mathrm{x}, \mathrm{y}$, and $\mathrm{z}$ axes, selection the trends for the new measurement plan, to determine relation of the dimensions to the mean differential parameters of the gradient. By splitting the measurement volume in such a manner, the coordinates of measurement will only depend on the characteristics of systematic errors. An implementation of this method in the measurement strategy is also discussed.

\section{Measurement of geometrical accuracy of the CMM}

One of the main steps used for the accuracy control of multi-coordinate machines, robots and measuring equipment is to calibrate the accuracy of geometric elements of the machine - the straightness, perpendicularity, flatness of guide ways and bedding; the bias of vertical, horizontal and longitudinal traverse, pitch, roll, yaw, etc. This depends on necessity to create the system of datum to assure the highest accuracy of operation of the machine in the volume with six degrees of freedom.

The geometric errors in the volume of a multicoordinate machine consist (Fig 1a) of the perpendicularity of coordinate axes $\Delta_{x / y}, \Delta_{x / z}, \Delta_{y / z}$, the coordinate position errors $\Delta_{x, y, z}$ along the axes $x, y$, and $z$; the rolling errors $\Delta \varphi_{x x} ; \Delta \varphi_{y y} ; \Delta \varphi_{z z}$ around the axes $x, y$ and $z$, yaw and pitch errors $\Delta \varphi_{x y} ; \Delta \varphi_{x z} ; \Delta \varphi_{y z} ; \Delta \varphi_{y x} ; \Delta \varphi_{z x} ; \Delta \varphi_{z y}$; lateral displacement errors $\Delta_{x(x y)} ; \Delta_{x(x z)} ; \Delta_{y(x y)} ; \Delta_{y(y z)} ; \Delta_{z(x z)} ; \Delta_{z(y z)}$ during the movement of the part along the relevant axis in the indicated plane ( $x y, z x$, etc.; the first of the indices shows the axis of movement). So, there are 21 type of geometric errors in 3D coordinate machines. Along with some other sampling strategies for determination of geometrical parameters, $L-P$ sequences method for this purpose was applied.

The aim of the $L-P$ sequences method [10] is to minimise the number of points and strokes of measurement during the coordinate and geometric error assessment of CMMs and to collect information no less than that in general cases. The method was used to find the points equally distributed in the working volume. Evenly distributed points according to the principle of $L-P$ sequences are shown in Fig 1 b.

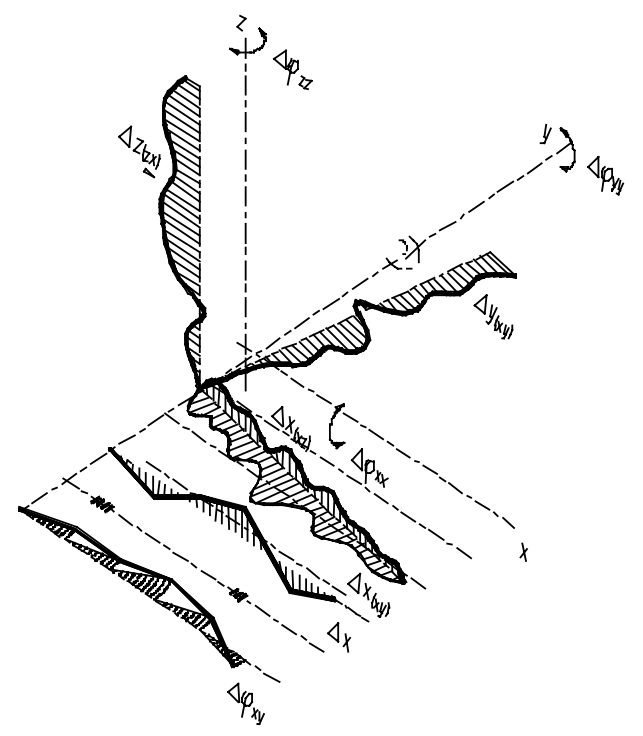

a)

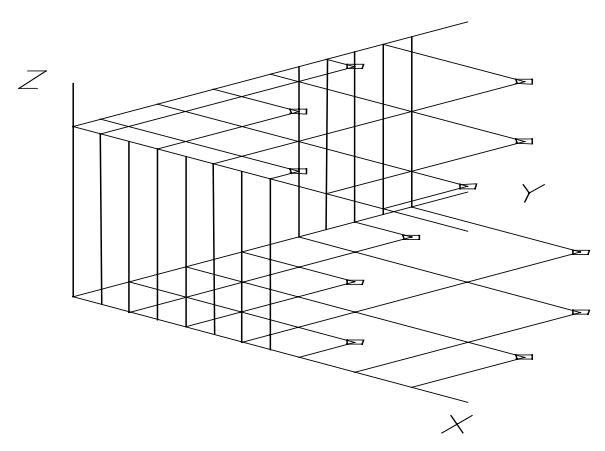

b)

Fig 1. Geometric errors distribution for machine product specification (a) and measuring points sampling in the measurement volume (b)

The points are most evenly distributed in the area when the number of points is

$$
N=2^{m}-1
$$

where $m=1,2, \ldots$

The coordinates of $n$-points in $L-P$ distribution are 


$$
Q_{i}=\left(q_{i 1}, \ldots, q_{i n}\right),
$$

$i=1,2, \ldots ; i$ - the index of a point. By writing the indices of points $i=e_{m}, \ldots e_{1}$ in the binary system and after performing a logic operation, it will become:

$$
q_{i j}=e_{1} v_{j} \cdot e_{2} v_{j}^{2^{*} \ldots{ }^{*}} e_{m} v_{j}^{m} .
$$

The operation designated by * performs the comparison of codes. The values of $v_{j}^{s}$ are taken from the tables whose size is $1 \leq s \leq 20,1 \leq j \leq 51$. Using $n \leq 51$, the $n$-dimensional $Q$ points will be found, $N \leq 2^{21}$.

The coordinates of points are calculated after determining $i$ for every number: $m=1+[\ln i / \ln 2]$. After that $j=1, \ldots, n$ is calculated.

The number of points can be chosen freely and depends on the dimensions of the CMM or other volume to be calibrated. An example of the points generated in such a way is presented in Fig 1b. According to the coordinates of points generated using the equations (1)(3), an experimental accuracy calibration of the machine was performed.

\section{Modified volume discretisation method}

A method for volume discretisation is proposed in $[6,9,10]$ where the pitch of the discretisation is calculated in inverse ratio to the values:

- of the gradient module:

$$
\begin{aligned}
& L_{\mathrm{int}}=q \frac{L}{|\bar{\Delta}|}, \\
& |\vec{\Delta}|=\sqrt{\left(\frac{\partial \Phi}{\partial x}\right)^{2}+\left(\frac{\partial \Phi}{\partial y}\right)^{2}+\left(\frac{\partial \Phi}{\partial z}\right)^{2}} ;
\end{aligned}
$$

- of the constituents of the gradient module:

$$
L_{\text {int } x}=q \cdot \frac{L}{\left|\frac{\partial \Phi}{\partial x}\right|}, \quad L_{\text {int } y}=q \cdot \frac{L}{\left|\frac{\partial \Phi}{\partial y}\right|}, \quad L_{\text {int } z}=q \cdot \frac{L}{\left|\frac{\partial \Phi}{\partial z}\right|},
$$

where $\Phi$ is an analytic expression of the local errors on the surface; $O x, O y, O z$ are coordinate axes; $L$ is the value of the pitch of discretisation of the surface by standard methods; and $q$ is the coefficient of proportion.

It must be noted that the value of partial derivatives is $\operatorname{tg} \alpha$, then it is valid to write: $\frac{\partial \Phi}{\partial x}=\operatorname{tg} \alpha_{x} ; \frac{\partial \Phi}{\partial y}=\operatorname{tg} \alpha_{y}$; $\frac{\partial \Phi}{\partial z}=\operatorname{tg} \alpha_{z}$, where the value of $|\vec{\Delta}|$ will be equal to some special calculated value of $\operatorname{tg} \bar{\alpha}$ :

$$
|\vec{\Delta}|=\sqrt{\left(\frac{\partial \Phi}{\partial x}\right)^{2}+\left(\frac{\partial \Phi}{\partial y}\right)^{2}+\left(\frac{\partial \Phi}{\partial z}\right)^{2}}=\operatorname{tg} \bar{\alpha} .
$$

The value of $\operatorname{tg} \alpha_{x}^{\prime}$ can be approximately determined according to Fig 2, by taking the adjacent values of $\Delta x$ :

$$
\operatorname{tg} \alpha_{x}^{\prime}=\frac{\Phi_{i+1}-\Phi_{x_{i}}}{x_{i+1}-x_{i}} .
$$

Calculating the values of $\operatorname{tg} \alpha_{x}^{\prime \prime}$ according to the adjacent values $\Delta x_{i+2}$ and $\Delta x_{i+1}$, it would be

$$
\operatorname{tg} \alpha_{x}^{\prime \prime}=\frac{\Phi_{i+2}-\Phi_{i+1}}{x_{i+2}-x_{i+1}},
$$

and it will yeld in $\operatorname{tg} \alpha_{x}^{\prime \prime}<\operatorname{tg} \alpha_{x}^{\prime}$.

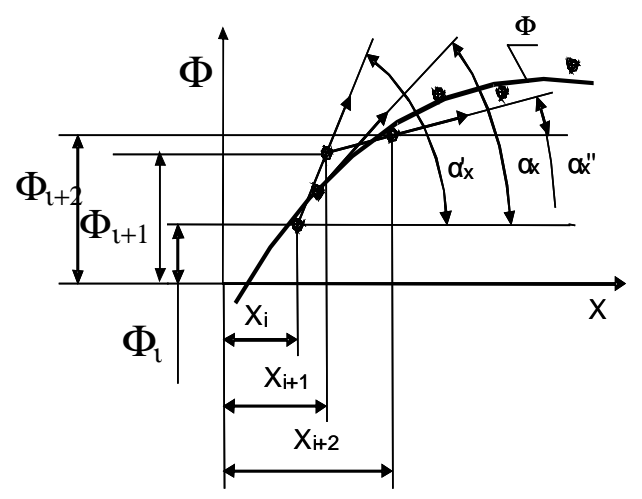

Fig 2. Graphical interpretation of measurement results

It is apparent from Fig 2 that by calculating the values of $\operatorname{tg} \alpha_{x}$, random errors will have a big influence on the result. They can appear in a little different way during the repeated measurements of the surface. The methods must be applied that permit to define the features of the gradient on the surface when more accurate evaluation of variation of the systematic errors is needed. Using the method of mathematical regression, the law of variation of the systematic error in local intervals $x_{i}, x_{i+1}$, $\ldots, x_{i+n}, y_{i}, y_{i+1}, \ldots, y_{i+n}$, and $z_{i}, z_{i+1}, \ldots, z_{i+n}$ can be determined by polynomials

- for the volume:

$$
\begin{aligned}
& \Phi=a_{1}+a_{2} x+a_{3} y+a_{4} z+a_{5} x^{2}+a_{6} y^{2}+a_{7} z^{2}+a_{8} x^{3}+ \\
& +a_{9} y^{3}+a_{10} z^{3}
\end{aligned}
$$

- for the plane:

$$
\Phi=a_{1}+a_{2} x+a_{3} y+a_{4} x^{2}+a_{5} y^{2}+a_{6} x^{3}+a_{7} y^{3} ;
$$

- for coordinate measurements:

$$
\Phi=a_{1}+a_{2} x+a_{3} x^{2}+a_{4} x^{3} .
$$

Here $a_{1}, a_{2}, \ldots a_{10}$ are the values of coefficients. A least square method must be used for determination of the variable coefficients $a_{1}, a_{2}, \ldots, a_{n}$ :

$$
A=\left(Y^{T} Y\right)^{-1} Y^{T} \bar{\Phi} \text {. }
$$

Here $A$ is a column of vector of the coefficients of variables $a_{1}, a_{2}, \ldots, a_{\mathrm{n}} ; Y$ is a rectangular matrix created according to method described in [6,9]; and $\Phi$ is 
a column of vector of the values of errors determined by experimental trials.

The expressions (9-12) show that each coordinate under different powers has three additives and one free member $a_{1}$. In this case, there will be 10,7 and 4 unknown members in the polynomials. Thus, for determination of their values there is a need to construct the same number of equations. Every equation will be created for a separate value of $\Phi_{i(i=1,2 \ldots)}$. The number of equations necessary for this purpose can be calculated in such a way:

$$
u=n+b,
$$

where $n$ is the number of unknown coefficients of the polynomial; $b \geq 1$ is freely chosen number showing the number of equations that will be created (more than unknown coefficients). Then the points $r_{x}, r_{y}$, and $r_{z}$ for each coordinate will be calculated by the expression

$$
r_{x}=r_{y}=r_{z}=r=u / p \text {. }
$$

Here $p$ is the different number of coordinates consisting in the calculation.

For the case discussed (Fig 2) five points must be used for assessing the systematic errors. By drawing the diagram of the third order $\Phi$ (Fig 2), the closest approach to the law of variation of the systematic error would be the least squares method. It must be noted that when using the latter method the filtering separates a random part of the errors. This helps to explain the consistency and adequate accuracy of the modified surface discretisation methods. This is the first main feature of its efficiency.

The second feature of efficiency of the modified surface discretisation method shows an ability of using this method to create the shortest length of the period of constituents of errors of industrials parts or the terrain of the area that can be evaluated by volume discretisation.

\section{Conclusions}

After performing an analysis of the effectiveness of determining the pitch of discretisation of the measurement surface or the volume by modified means, some conclusions can be made:

- The errors of the local surface and the characteristics of its gradient are more accurately determined by applying the methods of regression analysis;

- The dependence of the shortest length of period of constituents of errors on the pitch of measurement of the surface is determined. The means to improve effectiveness of measurement are pointed out;

- The methods of choosing the measuring strategy described can be successfully used for the machine engineering and surveying as well.

\section{References}

1. Kim W.-S.; Raman S. On the selection of flatness measurement points in coordinate measuring machine inspection. International Journal of Machine Tools and Manufacture, No 40, 2000, p. 427-443.

2. Lee G.; Mou J.; Shen Y. Sampling strategy design for dimensional measurement of geometric features using coordinate measuring machine. International Journal of Machine Tools and Manufacture, No 37(7), 1997, p. 917934.

3. Giniotis V. and Grattan K. T. V. Optical method for the calibration of raster scales, Measurement, No 32, 2002, p. 23-29.

4. Skeivalas J. Accuracy of geodetic coordinates determined by the pictures taken from air. Geodesy and cartography (Geodezija ir kartografija), No 27 (4), 2002, p. 131-134. (in Lithuanian).

5. Sladek J. and Krawczyk M. Modelling and assessment of large CMMs' accuracy. In: Proceedings, XVII IMEKO World Congress, Dubrovnik, Croatia, 2003, p. 1903-1906.

6. Mariūnas D., Giniotis V. Analysis of modified discretization methods for the measurement volume. Aviation, Vol 8, No 2, 2004, p. 21-24.

7. Giniotis, V.; Bansevičius, R.; Knight J. A. G. Complex accuracy assessment of multi-coordinate machines. In: Osanna et al ed.: Proceedings of the $6^{\text {th }}$ ISMQC IMEKO Symposium, Wien, Austria, 1998, p. 195-199.

8. Lin, Z. C.; Lin, W. S. Measurement point prediction of flatness geometric tolerance by using grey theory. Precision Engineering, No 25, 2001, p. 171-184.

9. Mariūnas, D. Integral modified discretization method of dimensional space. In: Proc. of XIV Int. Symp., Stralsund, Germany, May 5-8, 2004, p. 61-68.

10. Mariūnas, D. Modified of measurement space discretization method. Measurements (Matavimai), No 2 (26), Kaunas: Technologija, 2003, p. 36-40.

11. Woo, T. C.; Liang, R.; Hsieg C. C. et al. Efficient sampling for surface measurement. Journal of Manufacturing Systems, No 14 (5), 1995, p. 345-354.

12. Yau, H.-T.; Menq, C-H. Automate CMM path planning for dimensional inspection of dies and molds having complex surfaces. International Journal of Machine Tools and Manufacture, No 35 (6), 1995, p. 861-876.

Vytautas GINIOTIS. Professor, Doctor Habil.

Vilnius Gediminas Technical University. Dept of Geodesy and Cadastre, Sauletekio al. 11, LT-10223 Vilnius-40, Lithuania (tel. +370 5 2744703, fax +370 5 2744705), e-mail: Vytautas.Giniotis@ai.vtu.lt.

Graduated from Kaunas Polytechnical Institute, Faculty of Mechanical Engineering, 1961. Engineer at various machine production factories; 1989-1994 - Scientific worker and Chief metrologist at Experimental Scientific Institute of Metal Cutting Machines (ENIMS) and in Vilnius State Grinding Machines factory. 1994-2003 - Head of the Department of Aviation Technologies at A. Gustaitis Aviation Institute of VGTU. From 2003 till now - Chief Scientific Worker in Institute of Geodesy, VGTU.

Member-expert of Lithuanian Science Council, member of editorial board of two scientific journals, have made presentations and taken part in many international scientific conferences, visited universities of England, Germany, Sweden, Italy, Austria. Author more than 160 scientific articles, including 3 booklets and 53 inventions/patents.

Darius MARIŪNAS. Doctoral student. Vilnius Gediminas Technical University, Dept of Geodesy and Cadastre. Saulètekio al. 11, LT-10223 Vilnius-40, Lithuania (tel. +37052744703), e-mail: gkk@ ap.vtu.lt.

Master degree in Mechanical Engineering, VGTU, 1995. Author and co-author of five research reports and seven papers.

Research interests: measurement information, performance of measurements by discretisation of measuring space. 